\title{
Avaliação da Fitotoxidez de Tecoma stans (L.) Kunth
}

\author{
Franciane Auxiliadora Cipriani ${ }^{1}$, Maria Auxiliadora Coelho Kaplan ${ }^{2}$, \\ Rosy Mary dos Santos Isaias ${ }^{3}$, Geraldo Luiz Gonçalves Soares ${ }^{4}$
}

\footnotetext{
${ }^{1}$ Departamento de Botânica, Museu Nacional, Universidade Federal do Rio de Janeiro - UFRJ, Rio de Janeiro/RJ, Brasil ${ }^{2}$ Núcleo de Pesquisas de Produtos Naturais, Universidade Federal do Rio de Janeiro - UFRJ, Rio de Janeiro/RJ, Brasil ${ }^{3}$ Departamento de Botânica, Instituto de Ciências Biológicas, Universidade Federal de Minas Gerias - UFMG, Belo Horizonte/MG, Brasil

${ }^{4}$ Departamento de Botânica, Instituto de Biociências, Universidade Federal do Rio Grande do Sul - UFRGS, Porto Alegre/RS, Brasil
}

\begin{abstract}
RESUMO
Tecoma stans foi introduzida no Brasil para fins ornamentais e ocorre tanto cultivada quanto subespontânea. Objetivando avaliar seu potencial alelopático, foi testado o efeito do extrato aquoso de suas folhas sobre a germinação e o desenvolvimento radicial de Lactuca sativa $\mathrm{L}$. (alface), Handroanthus ochraceus (Cham.) Mattos (ipê) e Medicago sativa L. (alfafa). O extrato causou uma forte inibição na germinação das sementes de alface. O desenvolvimento radicial de alface e alfafa foi reduzido em 75 e $30 \%$, respectivamente. Não foi observado efeito do extrato sobre a germinação ou o desenvolvimento radicial do ipê. Todas as espécies tiveram a velocidade de germinação reduzida. Observaram-se alterações micromorfológicas no ápice radicial das plântulas de alface.
\end{abstract}

Palavras-chave: potencial alelopático, germinação, bignoniaceae.

\section{Evaluation of the Phytotoxicity of Tecoma stans (L.) Kunth}

\begin{abstract}
Tecoma stans was introduced in Brazil for ornamental purposes and occurs both as a cultivated or sub-spontaneous plant. This study aims to evaluate the allelopathic potential of the aqueous leaf extract of Tecoma stans on the germination and root development of three plant species: Lactuca sativa L. (lettuce), Handroanthus ochraceus (Cham.) Mattos (ipe), and Medicago sativa L. (alfalfa). The aqueous leaf extract of Tecoma stans caused strong inhibition on lettuce germination. The seedlings of lettuce and alfalfa had their root development reduced by $75 \%$ and $30 \%$, respectively. There was no effect of the extract on the germination or root development of ipe. Also, the germination rate of the three species tested was reduced. Micromorphological changes were observed in the root apex of lettuce seedlings.
\end{abstract}

Keywords: allelopathic potential, germination, bignoniaceae. 


\section{INTRODUÇÃO}

De acordo com Rizvi \& Rizvi (1992), o conceito de alelopatia descreve a influência direta de um organismo sobre o outro, quer seja um prejuízo ou um benefício aparente, sendo tal efeito realizado por metabólitos, denominados aleloquímicos. Tais substâncias são produzidas por uma planta e liberadas no ambiente, tanto na fase aquosa do solo ou do substrato, quanto na atmosfera, ao redor das plantas vizinhas. A alelopatia é um processo ecológico importante em ecossistemas naturais e manejados, influenciando na sucessão vegetal primária e secundária, na estrutura, na composição e na dinâmica de comunidades vegetais nativas ou cultivadas (Chou, 1986; Rizvi \& Rizvi, 1992; Reigosa et al., 1999; Scrivanti et al., 2003).

Há milhares de substâncias conhecidas como alelopáticas, pertencentes a várias classes químicas, destacando-se os polifenóis (Rice, 1984; Almeida, 1988). O efeito alelopático dessas substâncias se refere à capacidade de inibir a germinação de sementes ou o desenvolvimento de outras espécies (Rodrigues \& Lopes, 2001). Muller (1969) sugere que os aleloquímicos podem desempenhar um papel significativo nos padrões vegetacionais e nas variações em microescala no ambiente químico do solo, tais como aquelas causadas pela decomposição de resíduos vegetais e pela liberação de metabólitos secundários, que seriam importantes para a germinação e o estabelecimento de sementes individuais.

Dentre os fatores decisivos para o sucesso da invasão de plantas exóticas, estão as alterações alelopáticas (Goslee et al., 2001). Apesar da importância do conhecimento sobre as espécies invasoras, poucos são os estudos que associam o potencial alelopático de uma planta com a sua capacidade de invadir e se estabelecer em novos ambientes. É importante ressaltar que a vegetação de uma determinada área pode ter um modelo de sucessão condicionado às plantas pré-existentes e às substâncias químicas que estas liberam para o meio ambiente (Rice, 1984; Almeida, 1988; Rizvi \& Rizvi, 1992). Logo, o impacto ambiental de uma espécie vegetal invasora pode ser estabelecido e avaliado através de análises químico-ecológicas.
Tecoma stans (L.) Kunth (Bignoniaceae) é uma arvoreta nativa do México e do sul dos Estados Unidos, usada no Brasil como planta ornamental, e ocorre como cultivada ou subespontânea por quase todo o território, o que lhe confere uma vasta amplitude ecológica. No norte do Paraná, essa bignoniácea é citada por causar danos às pastagens e ao meio ambiente. Tecoma stans já invadiu cerca de 50 mil hectares de pastagens desta região e encontrase em franco processo de expansão (Kranz \& Passini, 1997), sendo citada como uma das principais espécies invasoras no Brasil. Tentativas de controle por meio da integração de métodos químicos e práticas culturais foram executadas sem sucesso (Kranz \& Passini, 1997; Passini \& Kranz, 1997; Ziller, 2001).

Apesar de possuir substâncias potencialmente alelopáticas, tais como flavonoides, taninos, alcaloides, quinonas e iridoides (Harborne, 1967; Rohatgi et al., 1983; Binutu \& Lajubutu, 1994; Von Poser et al., 2000), pouco se conhece sobre a ecologia química das espécies de Bignoniaceae.

O objetivo do presente estudo foi avaliar o potencial alelopático de T. stans sobre a germinação de Lactuca sativa L., Handroanthus ochraceus (Cham.) Mattos e Medicago sativa L.

\section{MATERIAL E MÉTODOS}

\subsection{Material vegetal}

Amostras de folhas de T. stans foram coletadas no Campus da Universidade Federal de Juiz de Fora, nos meses de novembro e dezembro de 2003. O Voucher encontra-se depositado no Herbário Padre Leopoldo Krieger da Universidade Federal de Juiz de Fora, sob o registro CESJ 14813.

\subsection{Teste de germinação}

A metodologia utilizada no presente trabalho foi baseada nos protocolos propostos por Rice (1984) e Soares et al. (2002). Para a obtenção dos extratos aquosos, folhas totalmente expandidas e sadias foram submetidas à maceração estática em água destilada à temperatura ambiente por $24 \mathrm{~h}$, obedecendo-se à proporção de 1:10 (material vegetal:água, p/v). 
Para detecção da fitotoxidez de T. stans, foi verificado o efeito de seu extrato aquoso sobre a germinação e o crescimento radicial de L. sativa (cv. 'Grand Rapids'), H. ochraceus e M. sativa (cv. crioula).

Utilizou-se um modelo totalmente aleatório para os ensaios de germinação, no qual amostras dos diásporos (alface e alfafa, 25 diásporos; ipê, 20 diásporos) foram distribuídas em Placas de Petri sobre papel filtro embebido com $5 \mathrm{~mL}$ do extrato. Água destilada foi usada como controle e cada tratamento foi realizado em cinco repetições. Os testes de germinação foram realizados à temperatura ambiente, que variou de 21 a $25^{\circ} \mathrm{C}$, e sob iluminação contínua fornecida por lâmpadas fluorescentes brancas de 20 watts.

Após três dias da semeadura, foi feita a contagem do número de sementes germinadas e medido o comprimento das raízes das plântulas de alface e alfafa, com o auxílio de um paquímetro. Para as sementes de ipê, a contagem do número de sementes germinadas e a medida do comprimento radicial foram realizadas sete dias após a semeadura.

Nos testes com alfafa e ipê, também foi avaliado o efeito dos extratos sobre a velocidade de germinação (IVG), segundo metodologia proposta por Labouriau (1983). Nos testes de índice de velocidade de germinação, foi incluído um controle osmótico equimolar aos extratos aquosos de T. stans, obtidos com uma solução de PEG 6000, segundo metodologia de Miro et al. (1998). Tendo como base esse trabalho, os valores de pressão osmótica obtidos para todos os extratos aquosos foram considerados baixos, variando ao redor de $-2,0$ bares. Deste modo, o controle osmótico foi usado apenas nos ensaios de velocidade de germinação, uma vez que ensaios preliminares (sem controle osmótico) demonstraram que esse era o único parâmetro afetado pelos tratamentos.

\subsection{Análise micromorfológica por Microscopia Eletrônica de Varredura (MEV)}

Os estudos em Microscopia Eletrônica de Varredura (MEV) foram realizados no Centro de Microscopia Eletrônica da Universidade Federal do Rio Grande do Sul (CME/UFRGS), em amostras de material fixado em FAA (Johansen, 1940). Raízes fixadas de alface foram desidratadas gradualmente em série acetônica (30\%, 50\%, 75\%, 90\% e 100\%) e secas em aparelho de ponto crítico Bal-tec ${ }^{\circledR}$ CPD 030. As amostras completamente secas foram afixadas aos porta-amostras ('stubs'), com auxílio de fita adesiva dupla face, e então metalizadas com uma camada de $20 \mathrm{~nm}$ de ouro, em metalizador Balzers ${ }^{\circledR}$ mod. SCD 050. As amostras preparadas foram visualizadas ao microscópio eletrônico de varredura JEOL ${ }^{\circledR}$ JSM 5.800 .

\section{RESULTADOS E DISCUSSÃO}

As espécies tratadas com o extrato aquoso de $T$. stans apresentaram alterações marcantes na percentagem de germinação (G\%) (Figura 1); enquanto o extrato de T. stans inibiu em $98 \%$ a germinação das sementes de alface $(\mathrm{G} \%=2)$, quando comparado ao grupo controle $(\mathrm{G} \%=100)$, o mesmo não ocorreu com as demais sementes das espécies testadas. Para as sementes de alfafa tratadas com o extrato de T. stans, a germinação foi de $76 \%$, enquanto o grupo controle apresentou $\mathrm{G} \%=85$; as sementes de ipê, por sua vez, apresentaram germinação estatisticamente semelhante à do grupo controle $(\mathrm{G} \%=80)$.

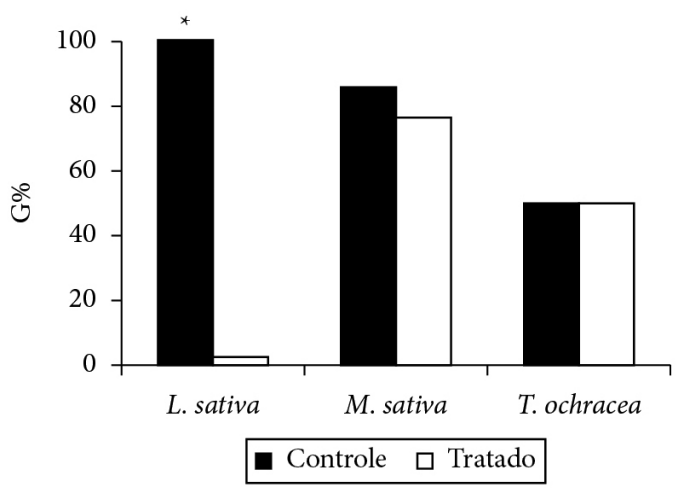

Figura 1. Efeito do extrato aquoso de Tecoma stans sobre a germinabilidade de Lactuca sativa, Medicago sativa cv. crioula e Handroanthus ochraceus. Valores assinalados com asterisco $\left(^{*}\right)$ diferem do grupo-controle, pelo teste de Tukey a $5 \%$.

Figure 1. Effect of the aqueous extract of Tecoma stans on the germinability of Medicago sativa cv. crioula and Handroanthus ochraceus). Values marked with asterisk $\left.{ }^{\star}\right)$ differ from the control group by the Tukey's test at $5 \%$. 
O efeito acentuado sobre a alface confirma essa espécie-alvo como uma das mais sensíveis a fitotoxinas. Por outro lado, os resultados com as demais espécies-alvo, alfafa e ipê, revelam um padrão observado por diversos autores, segundo o qual o processo de germinação é menos afetado por substâncias potencialmente alelopáticas (Ferreira, 2004).

Os compostos alelopáticos influenciam diretamente no desenvolvimento da raiz primária das espécies-alvo em ensaios de fitotoxidez, pois frequentemente interferem na divisão celular, na permeabilidade das membranas e na ativação das enzimas (Rodrigues et al., 1992; SchimidtSilva et al., 2011; Pawlowski et al., 2012). Em relação ao efeito sobre o desenvolvimento inicial da raiz primária das plântulas, nem todas as espécies testadas apresentaram redução do comprimento radicial (CR), quando comparadas aos respectivos grupos-controle (Figura 2). Plântulas de alface tratadas com o extrato de T. stans apresentaram um $\mathrm{CR}=0,40 \mathrm{~cm}$, o que significou uma redução média de aproximadamente $75 \%$ no desenvolvimento radicial, quando comparada com o respectivo controle. Nas plântulas de alfafa, a redução do CR foi de $27 \%$. As plântulas de ipê tratadas com o extrato de T. stans apresentaram alteração do desenvolvimento

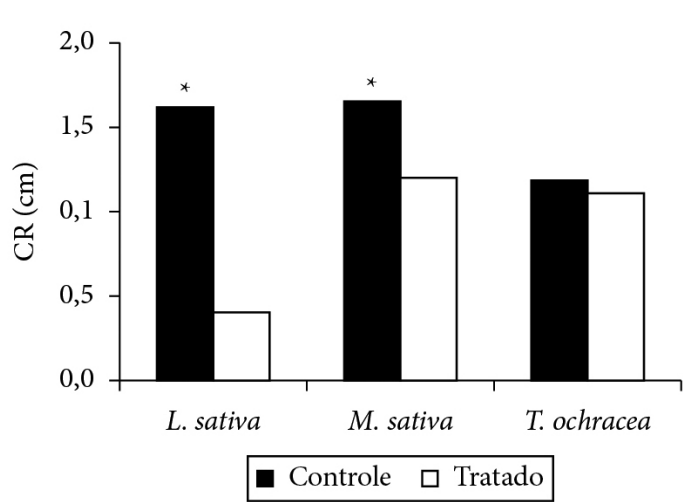

Figura 2. Efeito do extrato aquoso de Tecoma stans sobre o crescimento das raízes de Lactuca sativa, Medicago sativa cv. crioula e Handroanthus ochraceus. Valores assinalados com asterisco $\left(^{*}\right)$ diferem entre si pelo teste de Tukey a $5 \%$.

Figure 2. Effect of the aqueous extract of Tecoma stans on the root growth of Lactuca sativa, Medicago sativa cv. crioula and Handroanthus ochraceus). Values marked with asterisk $\left(^{\star}\right)$ by differ from the control by the Tukey's test at 5\%. radicial, apesar de a mesma, estatisticamente, não ser significativa.

$O$ índice de velocidade de germinação (IVG) é usado para avaliar o vigor das sementes, o qual é definido como a propriedade da semente que determina o potencial para uma emergência rápida e uniforme, e para o desenvolvimento de plântulas normais (Piña-Rodrigues et al., 2004). Apesar do reduzido efeito sobre a porcentagem de germinação final e sobre o desenvolvimento radicial da alfafa e do ipê, o extrato de $T$. stans retardou a velocidade de germinação dessas sementes (Figura 3). O tempo médio de germinação, em relação aos controles, foi reduzido em $46,22 \%$ nas sementes de alfafa e em 5,19\% nas sementes de ipê. Wardle et al. (1991) estudaram o potencial alelopático de Carduus mutans L. sobre espécies forrageiras e concluíram que houve inibição na germinação e no alongamento da radícula. Os autores, contudo, afirmaram ser a velocidade de germinação o indicador mais sensível do potencial alelopático.

Outro efeito causado por substâncias alelopáticas é o aparecimento de plântulas anormais, sendo a necrose da radícula um dos sintomas mais comuns (Ferreira \& Aquila, 2000). A análise
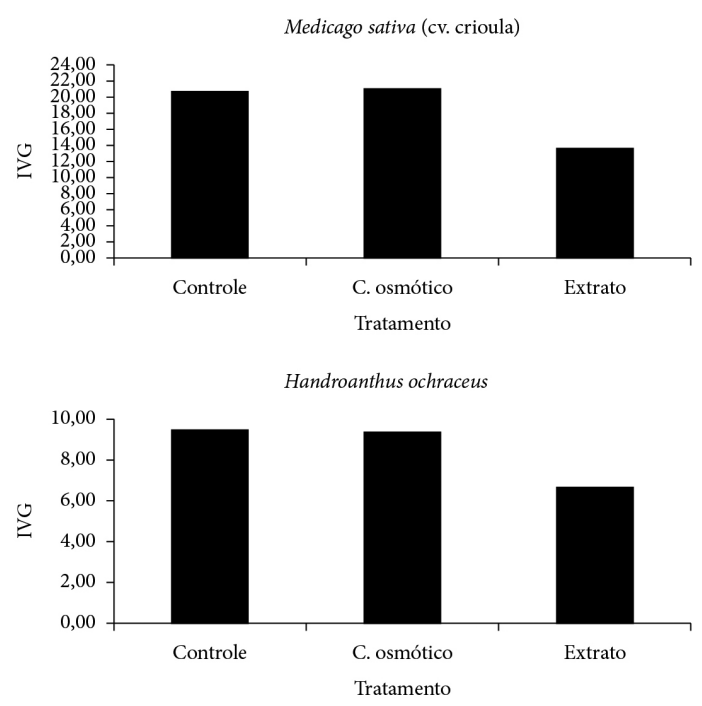

Figura 3. Efeito do extrato aquoso de Tecoma stans sobre a velocidade de germinação de Medicago sativa cv. crioula e Handroanthus ochraceus.

Figure 3. Effect of the aqueous extract of Tecoma stans on the speed of germination of Medicago sativa cv. crioula and Handroanthus ochraceus. 
micromorfológica por Microscopia Eletrônica de Varredura permitiu a detecção de diversas anomalias nas plântulas de alface tratadas com o extrato de T. stans (Figura 4). Enquanto as plântulas-controle apresentavam pilosidade relativamente baixa, as plântulas tratadas apresentavam alta densidade de pelos absorventes. Outro aspecto que pôde ser observado é o engrossamento do eixo radicial, principalmente na região pilífera. Bioensaios de crescimento com extratos aquosos de Cecropia pachystachya Trec. e Sapium glandulatum (Vell.) Pax aplicados à alface também permitiram visualizar maior densidade de pelos nas raízes das plântulas, bem como o espessamento dessas (Maraschin-Silva \& Aqüila, 2006). As plântulas tratadas de alface, alfafa e ipê também exibiram uma grande redução da coifa. Essa estrutura era nítida nos controles, caracterizada por células da região do ápice radicial com paredes anticlinais engrossadas. Em muitos ápices radiciais
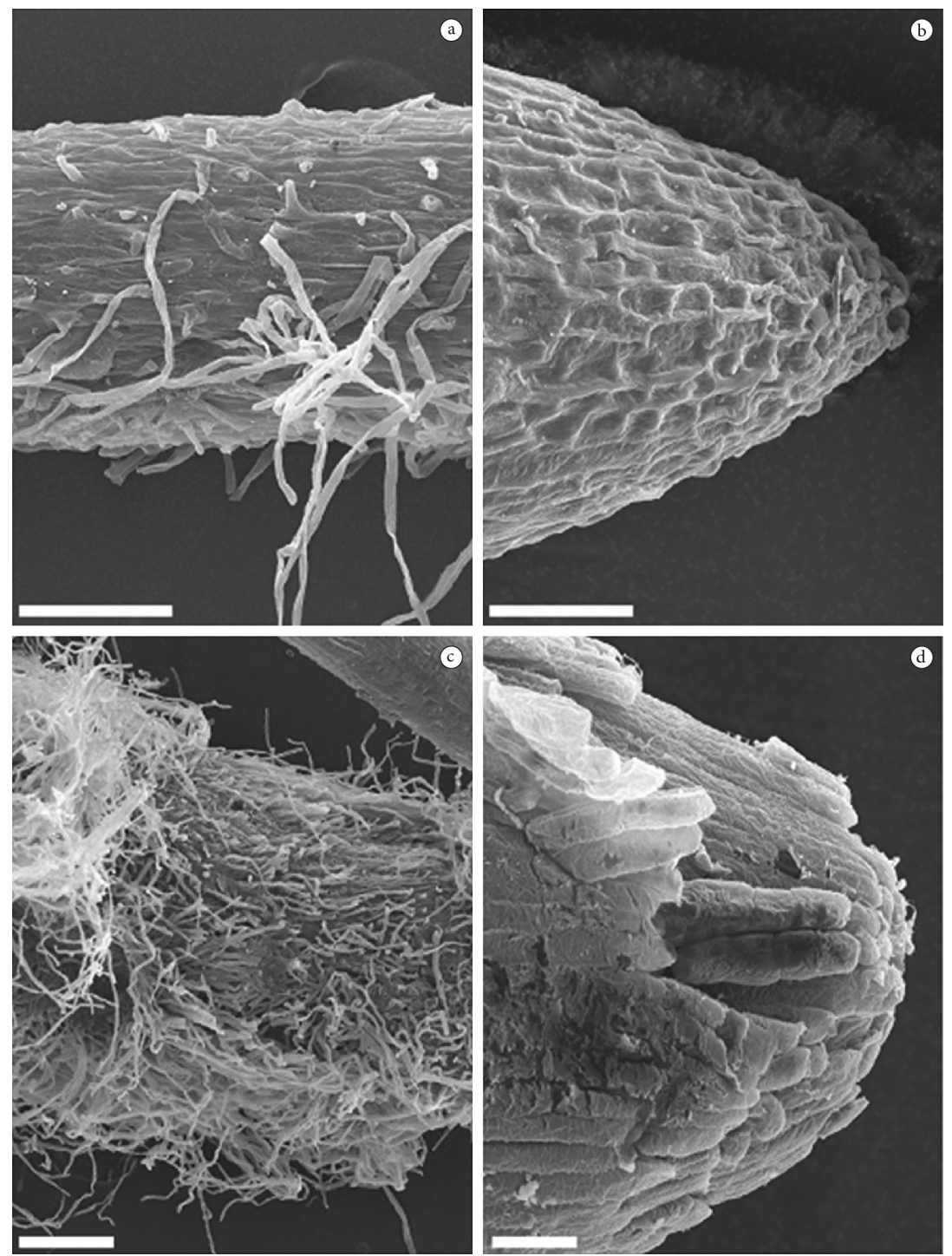

Figura 4. Aspectos micromorfológicos de plântulas de alface (Lactuca sativa cv. 'Grand Rapids'). a: região pilífera da raiz de uma plântula-controle; b: ápice radicial de uma plântula-controle; c: região pilífera da raiz de uma plântula tratada; d: ápice radicial de uma plântula tratada. Barra $=100 \mathrm{~mm}$.

Figure 4. Micromorphological view of lettuce seedlings (Lactuca sativa cv. "Grand Rapids"). a: piliferous zone of control seedling root; b: root apex of control seedling; c: piliferous zone of treated seedling root; d: root apex of treated seedling. Bar $=100 \mathrm{~mm}$. 
de plântulas tratadas com o extrato aquoso de $T$. stans, a coifa estava aparentemente ausente.

Análises fitoquímicas realizadas com folhas de $T$. stans demonstraram a presença de vários metabólitos secundários, como iridoides carbocíclicos e alcaloides derivados da via do ácido acético, incluindo alcaloides iridoídicos e derivados do ácido benzoico (Bianco et al., 1981, 1982; Constantino et al., 2003; Gross et al., 1973; Kunapuli \& Vaidyanathan, 1984; Lins \& Felicio, 1993; Mohan et al., 1979; Nair \& Vaidyanathan, 1964). Em princípio, os derivados do ácido benzoico parecem ser os principais candidatos a substâncias fitotóxicas presentes no extrato aquoso de T. stans.

Por outro lado, estudos recentes de Cipriani et al. (2010, 2012) com a tribo Tecomeae, revelam que iridoides carbocíclicos e alcaloides iridoídicos são apresentados como os principais marcadores das espécies deste táxon. Tal informação pode nortear a busca por outras possíveis substâncias alelopáticas presentes no extrato aquoso de T. stans. De fato, já foram descritos efeitos fitotóxicos para derivados iridoídicos glicosilados e potencialmente hidrossolúveis (Tundis et al., 2008).

\section{CONCLUSÕES}

Os resultados deste trabalho confirmam a fitotoxidez do extrato de T. stans. Houve uma clara variação do efeito entre as espécies testadas, sendo a alface a espécie-alvo mais sensível dentre as três espécies testadas, o que foi confirmado pelo aparecimento de alterações micromorfológicas apenas nas raízes dessa espécie.

\section{STATUS DA SUBMISSÃO}

Recebido: $26 / 04 / 2013$

Aceito: 08/11/2013

Publicado: 31/02/2014

\section{AUTOR(ES) PARA CORRESPONDÊNCIA}

\section{Franciane Auxiliadora Cipriani}

Departamento de Botânica, Museu Nacional, Universidade Federal do Rio de Janeiro - UFRJ, CEP 20940-040, Rio de Janeiro, RJ, Brasil e-mail: francipriani@gmail.com

\section{APOIO FINANCEIRO}

$\mathrm{CNPq}$

\section{REFERÊNCIAS}

Almeida FS. A alelopatia e as plantas. Londrina: IAPAR; 1988.

Bianco A, Massa M, Oguakwa JU, Passacantilli P. 5-deoxystansioside, na iridoid glucoside from Tecoma stans. Phytochemistry 1981; 20(8): 1871-1872. http:// dx.doi.org/10.1016/0031-9422(81)84024-1

Bianco A, Guiso M, Iavarone C, Massa M, Trogolo C, Oguakwa JU et al. Isolation of stansioside, a new iridoid glucoside from Tecoma stans, and reassignment of the stereochemistry of the $\mathrm{C}(8)$ center of tecomoside. Gazzetta Chimica Italiana 1982; 112(5-6): 199-203.

Binutu OA, Lajubutu BA. Antimicrobial potentials of some plant species of Bignoniaceae family. South African Journal of Medical Sciences 1994; 23(3): 269-273.

Chou $\mathrm{CH}$. The role of allelopathy in subtropical agroecosystems in Taiwan. In: Putnam AL, Tang CS, editores. The science of allelopathy. New York: John Wiley \& Sons; 1986.

Cipriani FA, Cidade WF, Soares GLG, Kaplan MAC. Estudo da similaridade química em tribos de Bignoniaceae Juss. In: Kaplan MAC, Abreu HS, Lima HRP, Soares GLG, editores. Abordagem quimiossistemática e evolução química de fanerógamas. Seropédica: Edur; 2010.

Cipriani FA, Figueiredo MR, Soares GLS, Kaplan MAC. Implicações químicas na sistemática e filogenia de Bignoniaceae. Química Nova 2012; 35(11): 2125-2131. http://dx.doi.org/10.1590/S0100-40422012001100005

Constantino L, Raimondi L, Pirisino R, Brunetti T, Pessotto P, Giannessi $\mathrm{F}$ et al. Isolation and pharmacological activities of Tecoma stans alkaloids. Farmaco 2003; 58(9): 781-785. http://dx.doi. org/10.1016/S0014-827X(03)00133-2

Ferreira AG. Interferência: competição e alelopatia. In: Ferreira AG, Borghetti F, editores. Germinação: do básico ao aplicado. Porto Alegre: Ed. Artmed; 2004.

Ferreira AG, Aquila MEA. Alelopatia: uma área emergente da ecofisiologia. Revista Brasileira de Fisiologia Vegetal 2000; 12: 175-204.

Goslee SC, Peters DPC, Beck KG. Modeling invasive weeds in grasslands: the role of allelopathy in Acroptilon repens invasion. Ecological Modelling 2001; 139(1): 31 45. http://dx.doi.org/10.1016/S0304-3800(01)00231-9

Gross D, Berg W, Schütte HR. D 5 -Dehydroskytanthin and d-skytanthin in Tecoma stans. Phytochemistry 
1973; 12(1): 201-202. http://dx.doi.org/10.1016/S00319422(00)84648-8

Harborne JB. Comparative biochemistry of the flavonoids-VI. Flavonoid patterns in the Bignoniaceae and the Gesneriaceae. Phytochemistry 1967; 6(12): 16431651. http://dx.doi.org/10.1016/S0031-9422(00)828976

Johansen DA. Plant Microtechnique. New York: McGraw-Hill Book; 1940.

Kranz WM, Passini T. Amarelinho: biologia e controle. Londrina: IAPAR; 1997.

Kunapuli S, Vaidyanathan CS. Indolic compounds in the leaves of Tecoma stans. Phytochemistry 1984; 23(8): 1826-1827. http://dx.doi.org/10.1016/S00319422(00)83513-X

Labouriau LG. A germinação das sementes. Washington: Departamento de Assuntos Científicos e Tecnológicos da Secretaria Geral da Organização dos Estados Americanos; 1983

Lins AP, Felicio JD. Monoterpene alkaloids from Tecoma stans. Phytochemistry 1993; 34(3): 876-878. http://dx.doi.org/10.1016/0031-9422(93)85381-Z

Maraschin-Silva F, Aqüila MEA. Potencial alelopático de espécies nativas na germinação e crescimento inicial de Lactuca sativa L. (Asteraceae). Acta Botanica Brasilica 2006; 20(1): 61-69. http://dx.doi.org/10.1590/ S0102-33062006000100007

Miro CP, Ferreira AG, Aquila MEA. Alelopatia de frutos de erva-mate (Ilex paraguariensis) no desenvolvimento do milho. Pesquisa Agropecuária Brasileira 1998; 33(8): 1261-1270.

Mohan VP, Kishore G, Sugumaran M, Vaidyanathan CS. Purification and properties of protocatechuate-3,4dioxygenase from Tecoma stans L. Plant Science Letters 1979; 16(2-3): 267-272. http://dx.doi.org/10.1016/03044211(79)90037-3

Muller CM. Allelopathy as a factor in ecological process. Vegetatio 1969; 18: 348-357. http://dx.doi.org/10.1007/ BF00332847

Nair PM, Vaidyanathan CS. An indole oxidase isolated from the leaves of Tecoma stans. Biochimica et Biophysica Acta 1964; 81(3): 496-506. PMid:14170321.

Passini T, Kranz WM. Eficácia de herbicidas no controle de Amarelinho (Tecoma stans) em pastagem. Planta Daninha 1997; 15(2): 190-197. http://dx.doi. org/10.1590/S0100-83581997000200012

Pawlowski A, Kaltchuk-Santos E, Zini CA, Camarão EB, Soares GLG. Essential oils of Schinus terebinthifolius and S. molle (Anacardiaceae): Mitodrepessive and aneugenic inducers in onion and lettuce root meristems. South African Journal of Botany 2012; 80: 96-103. http:// dx.doi.org/10.1016/j.sajb.2012.03.003
Piña-Rodriguez FCM, Figliolia MB, Peixoto MC. Testes de Qualidade. In: Ferreira AG, Borghetti F, editores. Germinação: do básico ao aplicado. Porto Alegre: Ed. Artmed; 2004.

Reigosa MJ, Sánchez-Moreiras A, González L. Ecophysiological approach in allelopathy. Critical Reviews in Plant Sciences 1999; 18(5): 577-608. http:// dx.doi.org/10.1016/S0735-2689(99)00392-5

Rice EL. Allelopathy. 2nd ed. Orlando: Academic Press; 1984.

Rizvi SJH, Rizvi V. Allelopathy Basic and Applied Aspects. London: Chapman \& Hall; 1992.

Rodrigues FCMP, Lopes BM. Potencial alelopático de Mimosa caesalpinaefolia Benthsobre sementes de Tabebuia alba (Cham). Sandw. Floresta e Ambiente 2001; 8(1): 130-136.

Rodrigues LRA, Rodrigues TJD, Reis RA. Alelopatia em plantas forrageiras. Jaboticabal: FCAVJ-UNESP; 1992.

Rohatgi BK, Gupta RB, Roy D, Khanna RN. Quinones from Tecoma pentaphylla: Constitution of Tecomaquinones I and II. Indian Journal of Chemistry. Section B 1983; 22(9): 886-889.

Schimidt-Silva V, Pawlowski A, Santos EK, Zini CA, Soares GLG. Cytotoxicity of essencial oils from two species of Heterothalamus (Asteraceae). Australian Journal of Botany 2011; 59: 681-690.

Scrivanti LR, Zunnino MP, Zygadlo JA. Tagetes minuta and Schinus areira essential oils as allelopathic agents. Biochemical Systematics and Ecology 2003; 31: 563-572. http://dx.doi.org/10.1016/S0305-1978(02)00202-8

Soares GLG, Scalon VR, Pereira TO, Vieira DA. Potencial alelopático do extrato aquoso de folhas de algumas leguminosas arbóreas brasileiras. Floresta e Ambiente 2002; 9(1): 119-126.

Tundis R, Loizzo MR, Menichini F, Statti GA, Menichini F. Biological and pharmacological activities of iridoids: recent developments. Mini Reviews in Medicinal Chemistry 2008; 8(4): 399-420. PMid:18473930. http:// dx.doi.org/10.2174/138955708783955926

Von Poser GL, Schripsema J, Henriques AT, Jensen SR. The distribution of iridoids in Bignoniaceae. Biochemical Systematics and Ecology 2000; 28(4): 351366. http://dx.doi.org/10.1016/S0305-1978(99)00076-9

Wardle DA, Ahmed M, Nicholson KS. Allelopathic influence of nodding thistle (Carduus nutans) seeds on germination and radicle growth of pasture plants. New Zealand Journal of Agricultural Research 1991; 34(2): 185-191. http://dx.doi.org/10.1080/00288233.1991.104 23358

Ziller SR. Plantas exóticas invasoras: a ameaça da contaminação biológica. Ciência Hoje 2001; 30(178): 77-79. 